\title{
Metanoia económica desde la complejidad
}

\section{Economic metanoia from complexity}

Recibido: $15 / 08 / 2020$

Aceptado: 15/12/2020
Oscar Eduardo Orellana Aldana Axel Esaú Brenes Villeda² Adery Luciano Pérez Yoc $^{3}$ Sergio Giovanni Gatica ${ }^{4}$ Correo: jutebek52@gmail.com Filiación de los autores: Universidad de San Carlos de Guatemala https://doi.org/10.36314/revistavida.v2i1.7

\section{Resumen}

Metanoia económica, surge precisamente de la necesidad de analizar nuestro contexto actual y de brindarle respuestas a un ecosistema que hoy más que nunca tiene muchas interrogantes sobre el futuro que deviene a partir de la pandemia COVID-19. Seguramente los protagonistas de este futuro seguirán siendo los mismos, consumidores, productores y gobierno, pero su comportamiento ante el uso de los recursos que nos provee el planeta, debe ser diferente. Caer en la misma panacea universal para resolver los problemas, como hasta el momento ha sido el capitalismo, mediante la propensión del crecimiento económico a costa de la destrucción del planeta, en la práctica ya no es una respuesta confiable; se debe precisar un cambio desde adentro, una conversión que conduzca a los protagonistas a la defensa de la vida en toda su expresión y como resultado al biocomportamiento.

En este sentido, surge la necesidad de recurrir a la bioeconomía, y en consecuencia emerge la biotributación como una opción, para desincentivar la inversión, fomentar el consumo responsable y la tributación verde o ecotributación, para la vida y su conservación. Las biofinanzas por su parte, buscan la correcta administración de los biorecursos que son obtenidos de bioactividades; para ello se apoya en las neurofinanzas, con el propósito de entender la racionalidad clásica del ser humano y enfocarla en una nueva racionalidad en la toma de sus decisiones.

Gracias a la creatividad humana y a su fundamental herramienta, la cultura, en donde se incluye tanto a la ciencia como al arte, la civilización actual ha alcanzado elevados niveles de confort. En lo inmanente y material. Todo (quizá) estaría bien a no ser porque en lo espiritual, en lo ético, en lo trascendente como especie y no como individuos literalmente hemos devastado al planeta Tierra. Hemos alcanzado un punto de inflexión que como especie estamos al borde de la extinción y por ende debemos encontrar una solución.

\section{Palabras clave}

Metanoia, Agentes económicos, Autopoiesis, Biocomportamiento, Bioeconomía, Bioética, Biofinanzas, Biotributos, COVID-19, Ecosistema, Holograma, Recursividad

1 Licenciado en Administración de Empresas, M. Sc. en Administración de Negocios.

2 Contador Público y Auditor, Máster en Hacienda Pública y Administración Financiera y Tributaria.

3 Licenciado en Administración de Empresas, M. Sc. en Gerencia de Recursos Humanos y M.A. en Gerencia de Mercadeo Estratégico.

4 Ingeniero Industrial, Maestro en Administración Financiera. 


\begin{abstract}
Abstrac
Economic methanoia arises precisely from the need to analyze our current context and provide answers to an ecosystem that today more than ever has many questions about the future that comes from the COVID-19 pandemic. Surely the protagonists of this future will continue to be the same, consumers, producers and government, but their behavior towards the use of the resources provided by the planet must be different. Falling into the same universal panacea to solve problems, as capitalism has hitherto been, through the propensity for economic growth at the cost of destroying the planet, in practice is no longer a reliable response; a change must be needed from within, a conversion that leads the protagonists to the defense of life in all its expression and as a result to biobehavior.

In this sense, the need arises to resort to the bioeconomy, and consequently bi-taxation emerges as an option, to discourage investment, promote responsible consumption and green taxation or ecotribución, for life and its conservation. For their part, biofinance seeks the correct administration of bioresources that are obtained from bioactivities; To do this, it relies on neurofinance, in order to understand the classic rationality of the human being and focus it on a new rationality in decision making.

Thanks to human creativity and its fundamental tool, culture, which includes both science and art, today's civilization has reached high levels of comfort. In the immanent and material. Everything (perhaps) would be fine if not because in the spiritual, in the ethical, in the transcendent as a species and not as individuals we have literally devastated planet Earth. We have reached a turning point that as a species we are on the verge of extinction and therefore we must find a solution.
\end{abstract}

\title{
Keywords
}

Economic agents, Autopoiesis, Biobehavior, Bioeconomy, Bioethics, Biofinance, Biotributos, COVID-19, Ecosystem, Hologram, Recursion

\section{Comportamiento complejo de los agentes económicos}

Desde hace varios siglos hemos vivido bajo el viejo paradigma del crecimiento económico, atados a una era del hiperconsumismo que se ha visto como la única solución para realizar la utópica tarea de mantener ocupada y entretenida a las masas, mientras nuestros recursos naturales perecen a merced de la producción desmedida provocada por la atroz, voraz y frenética ambición de la mayoría de corporaciones, dueñas de la producción de bienes y servicios en el mundo. De algún modo, los acontecimientos provocados por la pandemia del COVID-19, han despertado el deseo transigente de encontrar una nueva forma de vivir, que no dependa particularmente del crecimiento económico como la única alternativa para satisfacer las necesidades de la humanidad.

Hemos sido testigos de un panorama que ha obligado a las naciones más poderosas del mundo a adaptarse a cambios radicales en diferentes aspectos de la vida; el confinamiento, el cierre de fronteras, el uso de equipos de bioseguridad, las nuevas y duras condiciones económicas, pero sobre todo nos hemos adaptado a un mundo de pérdidas humanas (hijos, hermanos, padres, abuelos, esposos, parientes cercanos y lejanos, amigos, etc.), que ha provocado una gran 
ansiedad generada por la incertidumbre, alimentada incluso por las noticias falsas conocidas con el anglicismo de fake news de los medios de comunicación. Pareciera una película de ciencia ficción, sacada de la mente de los eruditos en la materia cineasta de Hollywood, únicamente que los actores en este drama si se enferman, si sufren, si mueren. En este vestíbulo de inseguridad, incertidumbre y desequilibrio, es preciso que los protagonistas asuman un papel principal y que el centro de esta conflagración sea precisamente en contra de la discriminación, desigualdad, miseria, pobreza, indiferencia, violencia, corrupción, enfermedades y tantos males que atañen la vida en este planeta, pero no únicamente la vida del ser humano, sino de todas las especies que habitan esta hermosa y compleja morada.

\section{El consumidor y las necesidades}

Previo a generar conclusiones sobre los objetivos e intencionalidad del presente artículo, es preciso esbozar un análisis del comportamiento histórico de cada uno de los protagonistas, que han procreado la plataforma caótica que en los últimos tiempos se manifiesta con una pandemia (COVID-19) y que reclama de manera intransigente los derechos de un planeta en decadencia, que busca por su propia cuenta recuperarse a toda costa de los impactos causados por el ser humano (homo economicus), que en realidad no es más que un homo sapiens controlado por sus emociones más profundas.

Todo parte de las necesidades del ser humano; de manera clásica podemos citar a Abraham Maslow, famoso psicólogo estadounidense conocido por ser pionero de la psicología humanista y que ha definido dentro de su teoría una pirámide de necesidades que plantea precisamente una jerarquía de las necesidades humanas.

Uno de los principios básicos que alimenta Maslow con su teoría, fundamenta que la base de la pirámide son necesidades innatas o intrínsecas del ser humano y son las fisiológicas y que representan la base de la pirámide, indicando que las demás nacen a partir de que se han suplido las necesidades básicas, por lo que usaremos el término consumidor, para las personas que satisfacen sus necesidades mediante la adquisición de bienes y servicios de distinta índole. De esa cuenta, el ser humano ya sea intrínseca o extrínsecamente se ve motivado a la búsqueda de bienes y servicios que contribuyan a satisfacer sus necesidades e incluso los deseos más ostentosos, no teniendo en cuenta el impacto perjuicioso que se produce en el medio ambiente de manera subyacente. 


\section{Las necesidades del productor}

Algo que es crucial en este análisis, es el hecho que para suplir la base de necesidades de la pirámide se solicitan recursos y los cuales el ser humano ha encontrado en la naturaleza misma, incluso en el proceso ha transformado los mismos, haciendo uso de su espíritu emprendedor. De tal suerte, que la ciencia económica clásica identifica estos recursos como factores de producción (trabajo, tierra y capital) los cuales se combinan para generar bienes y servicios que suplen las necesidades elementales de las familias; a este ente que se organiza para combinar adecuadamente dichos factores y generar los bienes y servicios, lo conocemos como productor, debido a que es el facilitador y proveedor de los mismos.

\section{Biocomportamiento}

De acuerdo con la tercera ley de Newton con toda acción ocurre siempre una reacción igual y contraria; según esta apreciación, se pensaría que si el ser humano tiene sed, mediante la consecución de tomar agua simplemente bastaría para cubrir con esa necesidad, una acción y una reacción a la vez; sin embargo, el asunto es más complejo, cuando el ser humano tiene sed no precisamente busca satisfacerla mediante la ingesta de agua, debido a que hoy existen tantas opciones que ofrece el mercado para una necesidad tan básica como la sed, de tal suerte, el consumidor puede elegir entre una bebida carbonatada y saborizada artificialmente, un jugo de verduras o frutas, una bebida alcohólica, entre tantas alternativas que los productores ofrecen en el mercado. Esto cambia rotundamente el comportamiento del consumidor, debido a que sus opciones ya no se basan en cubrir la necesidad, sino en llenar el deseo que ha sido creado previamente por el productor. La pregunta ante este fenómeno sería ¿qué pasaría si no existieran tantas opciones para satisfacer una necesidad tan básica y elemental como la sed?; la respuesta implicaría muchos efectos no lineales que intervienen desde la perspectiva de la complejidad. Algunos argumentos serían: menos factores empleados en la producción de las bebidas alternativas, por ende, una menor cantidad de recursos naturales explotados, disminuye la cantidad de desechos y desperdicios sólidos que fluyen a los océanos o que rondan en el ambiente; menores emisiones de gases de efecto invernadero que hoy en día son los causantes del cambio climático, menos problemas de salud en las personas como consecuencia de la reducción de azúcares, ingredientes artificiales y preservantes que se incluyen en dichos productos, entre otros.

El mismo ejemplo podría aplicarse para cualquier producto que satisfaga hoy en día alguna de las necesidades planteadas en la pirámide de Maslow, desde los bienes y servicios básicos como alimentación, agua y vestido, hasta los más sofisticados como salud, vivienda, transporte y comunicaciones; incluso, desde la perspectiva de las necesidades como el afecto y la amistad, hoy se ven divididas por clases sociales, según la cantidad de recursos económicos que los individuos po- 
sean; las relaciones sociales, la educación y el reconocimiento, hoy en día tienen tantos satisfactores, que las decisiones económicas cada vez son más complejas y el consumidor cada vez se vuelve más emocional al momento de tomarlas.

No obstante, el problema no existe precisamente en la complejidad de las decisiones del consumidor y en la obstinación del productor; si los recursos fueran ilimitados y los desechos no contaminaran el ambiente, no existiera problema alguno. Lamentablemente los recursos son cada vez más escasos y los niveles de destrucción de los recursos naturales y la contaminación han provocado estragos irreversibles en el ecosistema. Este ha sido uno de los agentes económicos que no se ha considerado como tal, simplemente ha estado al margen, excluido durante toda la historia; incluso, lo que ha generado una crisis a nivel global en materia ecológica, al nivel que los ambientalistas han previsto que si no se producen cambios drásticos en el comportamiento de los consumidores y productores, respecto al uso correcto de los recursos y el manejo adecuado de los desechos y emisiones de gases, antes del 2030, los daños al ecosistema debido al cambio climático serían catastróficos.

En este sentido, esto tendría un impacto en la salud, en la alimentación y en la subsistencia de muchas personas y especies de seres vivos en el planeta; lo que propicia una expectativa de vida muy limitada para las generaciones futuras y problemas drásticos con la sustentabilidad del planeta. Es un reto enorme para esta generación, encontrar las respuestas adecuadas a las grandes interrogantes respecto a la degradación ambiental y sus consecuencias, que deben incluir todas las conexiones posibles entre las diferentes disciplinas, pero principalmente las ciencias sociales como la economía, la sociología, las ciencias políticas, entre otras, que deberán promover un nivel de educación que promueva una conciencia diferente respecto a la relación con los sistemas vivos del planeta. "Una educación que privilegie la vida, es aquella que enseña a respetar los ciclos de los sistemas vivos, a valorar y apreciar a los otros seres y a entender que no podemos pensar únicamente en nuestro propio bienestar sino en el del planeta que habitamos." 5

La economía en todo su esplendor plantea modelos que facilitan al ser humano la administración de los recursos escasos, partiendo de que las necesidades son ilimitadas; resolver el problema de la escasez es uno de los argumentos que justifican el uso de los factores de producción (tierra, trabajo y capital) y lograr la eficiencia y eficacia en la producción, son reglas de oro que no se pueden quebrantar. Lamentablemente los modelos matemáticos de la economía con su famosa frase ceteris paribus analizan los problemas considerando que todo permanece constante y en un orden equilibrado, que nada cambia, que todo es igual, que ningún evento paralelo debe afectar su análisis.

5 Castillo López, Nancy Carolina, "El fenómeno del Coronavirus y el Biodesarrollo: sus consecuencias y oportunidades", Revista Ciencia Multidisciplinaria CUNORI Vol. 4, No. 2, USAC, Guatemala, 2008, Pág. 130. 
Sin embargo, la realidad es completamente diferente, nada es equilibrado, nada es constante, todo permanece en un cambio y en movimiento, desde el mundo cuántico en el que se expresan los fotones, neutrones, electrones y protones; hasta el mundo macroscópico, en el que se observa lo físico a simple vista, incluyendo el movimiento de los astros. De tal suerte, debemos comprender que, en este universo micro y macro lleno de incertidumbre, todo está conectado, que cualquier cambio que generemos en un sistema, afectará el ambiente de otro sistema, tanto aquellos sistemas que son macroscópicos, como aquellos que son microscópicos donde habitan incluso los virus. Naturalmente, podemos comprender y estimar incluso que lo que hoy vivimos como pandemia mundial, haya sido precisamente fruto de esos cambios obligados que el ser humano mediante su intervención ha realizado en el ambiente macro o micro; desde muchas perspectivas podemos apreciar que estos acontecimientos son efectos de autoorganización de los sistemas vivos, que al verse amenazados por fuerzas externas procuran como un mecanismo de defensa la activación de fenómenos naturales con la finalidad de continuar con su supervivencia, pero que de manera colateral han sido perjudiciales para el ser humano. ${ }^{6}$

Carlos Llarandi Arroyo en una publicación digital argumenta sobre los tres principios del pensamiento complejo, que pueden dar respuesta a muchos de los acontecimientos que en la actualidad nos tienen en una crisis sanitaria y económica, la cual sufrimos todos, desde los empresarios más acaudalados, hasta las personas marginadas de la sociedad, sin embargo todos buscamos respuestas, todos buscamos la verdad; ante este hecho Llarandi comenta: "La búsqueda de la verdad es una auténtica vocación del ser humano. El amor a la verdad nos permite acercarnos al concepto de bien y por tanto al bien común. La falta de coherencia absoluta, como la coherencia absoluta son dos enemigos de este proceso. El primero es evidente y nos lleva al sin sentido. Sin embargo, no hay que pensar que esto no es habitual; solo hay que mirar la política actual para darse cuenta de la irracionalidad. El segundo es un enemigo más sutil, por ser menos evidente. Puesto que nadie niega que la coherencia es algo bueno en sí mismo, absolutizarla puede llevar tanto a una simplificación inconsciente de la realidad (atajo, sesgo) como a un proceso interminable donde se intente encajar un puzle de infinitas piezas. Debemos aceptar humildemente la limitación del ser humano e intentar aprovechar al máximo nuestras capacidades. Autocrítica y revisión permanente son claves. ${ }^{7}$

En este sentido, Llarandi argumenta sobre el principio dialógico, el principio de recursividad y el principio hologramático. Por ejemplo, en economía el principio dialógico tiene dos aristas que son contradictorias, por un lado, el hecho de apro-

\footnotetext{
6 Rosa, Jeovany et al., "Dialogando con la Sociedad sobre Complejidad, Educación Salud y Vida", Revista Vida una Mirada Compleja Vol. 1, No. 1, p. 29. CUNORI. Guatemala, 2019.

7 Llarandi Arroyo, Carlos, "Tres principios del pensamiento complejo. La búsqueda de la verdad es una auténtica vocación del ser humano", Https://profesionalesporelbiencomun.com/tres-principios-del-pensamiento-complejo/\#.XrGo96hKjlu. Consulta realizada el 01 de mayo de 2020.
} 
vechar mediante la innovación tecnológica los recursos naturales, para satisfacer las necesidades humanas, no obstante, esto causa un impacto negativo fuerte en los ecosistemas incluso algunos de manera irreversible. Así también, el no hacer nada para para resolver los problemas económicos, pone en riesgo la vida de las personas, ese dilema ético se ha puesto de moda en la actualidad. Resguardar la vida y la salud de las personas, mediante el confinamiento, el aislamiento social y el cese de la producción; tienen una finalidad muy sensata de resguardar la vida, ante la pandemia del coronavirus, sin embargo, en un país como Guatemala, en el que personas viven de lo que logran producir en el día a día en la economía informal, parar la producción significa parar su sistema de subsistencia, también se pone en riesgo la vida y la estabilidad de este sector social. Estos dos hechos están correlacionados, por lo que debe buscarse básicamente aquello que está en medio, ese tercero que al momento es excluido y que puede unir a ambos o al menos dar una respuesta favorable ante las circunstancias.

Se entiende el principio de recursividad cuando el efecto influye en la causa que lo ha producido. Naturalmente, es bien conocido que en algunas ramas de la administración que plantean argumentos sobre calidad total, se sabe que los procesos de mejora continua se logran mediante los efectos positivos, cuya mejora preceden a otros efectos positivos, lo que genera un bucle positivo, que lleva a un concepto de eficiencia, eficacia y productividad en las empresas. La revolución tecnológica también es un ejemplo de bucle positivo, cuyos avances e innovación, han permitido el desarrollo de nuevas creaciones y así sucesivamente que establece un pronóstico de crecimiento y desarrollo tecnológico. Sin embargo, también podemos encontrar bucles negativos, los cuales provocan resultados a la inversa, un ejemplo muy claro de estos el mismo ser humano como especie, su crecimiento ha sido tan exitoso que ha provocado un bucle negativo respecto al ecosistema, a tal extremo que los recursos naturales se están extinguiendo exponencialmente, lo que pone incluso en riesgo la vida en toda su magnitud.

Para comprender de una forma holística lo que sucede en la actualidad, es importante recurrir a uno de los principios de la complejidad, el cual establece que no solo la parte está en el todo, sino que todo también está contenido en la parte. Lo que nos deja prácticamente con la inquietud de analizar a profundidad el comportamiento del ser humano tanto desde la perspectiva del consumidor, como desde la perspectiva del productor. Esto, para comprender la toma de decisiones económicas y por qué estas decisiones han tenido efectos antagónicos con el ambiente, que ponen en evidencia las debilidades del sistema económico que actualmente rige el mundo mediante la globalización y universalización sistemática. 


\section{El gobierno como tomador de decisiones}

Otra entidad que básicamente interviene en todo este proceso es el Estado, cosa pública que es administrada mediante un sistema de gobierno que debiera representar los intereses de la mayoría en la sociedad en un país. Esta entidad, se encarga de regular muchas de las transacciones que se realizan para que el productor y el consumidor tengan acceso a interactuar; cuyo propósito fundamental debería ser que este proceso se desarrolle de manera fácil, justa y ecuánime.

Sin caer en trivialidades, se ha descrito de manera sencilla la estructura que actualmente rige las transacciones económicas en el mundo, en la cual intervienen precisamente consumidores, productores y gobierno, a quienes en la ciencia clásica se les conoce como "agentes económicos". Lo que ahora nos atañe es desenmarañar el comportamiento actual de cada uno de estos agentes y las consecuencias positivas y negativas que produce cada una de las decisiones que asumen y que tienen repercusiones no lineales y complejas.

Los gobiernos, a pesar de que reciben para su operación tributos por parte de la sociedad, tanto de los consumidores como de los productores, no han sido capaces de administrar correctamente el impacto ecológico, las medidas que se han utilizado son lineales y precisamente han sido acciones correctivas, enfocadas en la reducción de las emisiones de efecto invernadero, por ejemplo; sin embargo, se han atacado los efectos y no las causas, las cuales por las características presentadas en este artículo son fenómenos de carácter complejo, no lineal, y que merecen un tratamiento transdisciplinar, holístico y al mismo tiempo hologramático, que permita visualizar en detalle los fenómenos y tomar decisiones radicales como las que propone nuestra intencionalidad "mejorar la calidad de vida con menos crecimiento económico".

\section{Bioeconomía y biotributación, para la vida y su conservación}

Al hilvanar, desde las ciencias clásicas, la relación entre Economía y tributación, ${ }^{8}$ resalta el término necesidades económicas, recordando que el hombre, desde el inicio de los tiempos, siente infinidad de necesidades, las cuales son satisfechas a través de bienes materiales o inmateriales (servicios), que sirven para satisfacer una necesidad humana.

Pues bien, entre los bienes y servicios económicos, se encuentran muchos imprescindibles para el bienestar de la sociedad: salud, educación, seguridad, vivienda, alimentación, carreteras, transporte, etcétera, y que para la satisfacción de los

8 Acotado a partir de información recogida en el documento: "Economía, Hacienda Pública y Política Fiscal. Tema 1: El Marco Económico Internacional. Macromagnitudes e Indicadores Macroeconómicos. Hacienda Pública y Fiscalidad: Teoría de la Imposición." José Manuel Guirola López y Javier Martín Román. Máster Oficial en Hacienda Pública y Administración Financiera y Tributaria de la Universidad Nacional de Educación a Distancia (UNED) y el Instituto de Estudios Fiscales (IEF). Tercera Edición 2017-2018. 
mismos interviene el sector público, mediante la detracción de recursos del resto de la economía, los cuales constituirán los ingresos públicos.

La forma más habitual, aunque no la única, de realizar esta detracción es mediante el establecimiento de tributos, categoría más importante de los ingresos fiscales de los Estados y que constituye el ingreso coactivo por excelencia.

Dado que los fines a los que los impuestos tratan de servir son cambiantes en el tiempo, con las circunstancias, con las ideologías, en definitiva, dependen de una decisión subjetiva, es decir, están circunscritos al campo de lo normativo -del deber ser- los principios impositivos también han sido a lo largo de la historia mutantes, discutiéndose, en función del momento, de las circunstancias o de la posición de cada uno, su validez e importancia.

Entre la clasificación de objetivos y principios impositivos, la mayoría de autores de Derecho Tributario, coinciden en que, entre los objetivos económicos de los tributos se tienen como principales: estabilidad económica, asignación de recursos, crecimiento económico y distribución justa de la renta y de la riqueza, por lo que resulta notoria la estrecha relación de la economía con la tributación, y al emerger desde las ciencias de la complejidad la bioeconomía, en consecuencia y en armonía, también emergería la biotributación, como una opción, para su consecución.

Respecto de la bioeconomía, Carlos Eduardo Maldonado indica que "La sociedad contemporánea ha elaborado al respecto un diagnóstico sólido, cuyos síntomas y signos, al mismo tiempo, son claros: hiperconsumo masivo, producción de productos de ciclos cortos de vida; esto es, la obsolescencia programada, la pérdida de la soberanía alimentaria, estilos y estándares de vida fundados en el tener, el gastar y haber-estado, agotamiento de los recursos naturales, producción en gran escala del efecto invernadero, acidificación de las aguas y los mares, son, entre muchos otros, algunos de los rasgos más sobresalientes de la decadencia y ulterior muerte de un sistema determinado. Pues bien, la bioeconomía pone el dedo en la llaga del sistema capitalista de producción y consumo gracias a que dirige la mirada del mercado y sus imperfecciones hacia la naturaleza y el medioambiente. El mercado no sabe para nada de entropía y sistemas alejados del equilibrio. La naturaleza es el ámbito en el que se dirime la gratificación de la vida, las ganas y el placer de vivir.?

De forma más ilustrativa y puntual, el mismo autor, también lo expresa en su ponencia "Bieconomía, ciencia y lenguaje", 10 en el que resalta que la economía se resume en macro, micro, finanzas y comercio y que los cuatro modelos (clásico,

9 Maldonado, Carlos Eduardo, "Complejidad de las ciencias sociales. Y de otras ciencias y disciplinas", Ediciones desde abajo. p. 202. Bogota, D.C., Colombia, 2016.

10 Canal de YouTube "Periódico desde abajo", https://www.youtube.com/watch?̨v=cMd44TPQ6XM. Consultado el 3 de mayo de 2020. 
neoclásico, economías de escala, desarrollo sostenible), constituyen una misma cosa, llámeseles antropológicos, antropomórficos o antropocéntricos, los que conciben al ser humano como superior a la naturaleza de la cual se ha apropiado y explotado.

Refiere que lo que ha permanecido intacto en el tiempo es un modelo de producción, que se traduce en hiperconsumo y agotamiento de recursos naturales.

Aborda el biodesarrollo, teniéndose éste como el núcleo de la bioeconomía, en función de la vida y no del mercado, con sistemas de exaltación, gratificación, posibilitamiento, en contraste con egoísmo e individualismo y por ende surgen principios de conciencia social, conciencia natural, lo que se traduciría en pensar en: bioeconomía, economía ecológica, ecología política, es decir en concreto o en resumen, pensar en el decrecimiento económico, que por los tiempos se ha traducido en un crecimiento puramente financiero. Esto implicaría, por ejemplo, sistemas de trueque y de consumo responsable, es decir como idea básica vivir mejor, vivir bien, pues el capitalismo es un sistema esquizofrénico que incluso hace desear el deseo.

Finalmente, propone una ecuación para el biodesarrollo, la cual, en términos comprensibles, consiste en multiplicar el crecimiento económico negativo (decrecimiento) por la potenciación (posibilitamiento, gratificación, exaltación) de la vida.

La decadencia de la economía pura o clásica se pone en relieve a raíz de la pandemia del Coronavirus (COVID-19), tal y como lo concluye provisionalmente el mismo autor ya referido: "La crisis del COVID-19 pone claramente sobre la mesa, a plena luz del día, un tema inocultable: debemos aprender a vivir; a vivir bien, a saber vivir. Alimentarse, bañarse las manos, saber qué consumir y porqué, saber qué dejar de consumir, y que la productividad, la competitividad y el crecimiento económico no son, en absoluto lo más importante. Sorpresivamente, la crisis del Conavid-19 le aporta todos los argumentos necesarios a las tesis del decrecimiento. Las sociedades complejas deben poder decrecer: la ecología política y la economía ecológica reciben, de contrapelo, toda la razón; en toda la línea de la palabra"."1

Podría intuirse que a hoy se tienen dos colapsos mundiales, uno económico y el otro sanitario, y ambos atentan contra la vida de muchas personas, vulnerables a contagiarse y con riesgo extremo de no lograr superar la enfermedad y/o de personas que no logren obtener los recursos necesarios y básicos para su subsistencia y supervivencia. De cualquier forma, se asemeja a una ruleta rusa en la cual está en juego la vida del ser humano.

11 Maldonado, Carlos Eduardo, "¿Qué significa la crisis del Coronavirus?", https://www.desdeabajo.info/ediciones/item/39464-que-significa-la-crisis-del-coronavirus.html. Consultado el 3 de mayo de 2020. 
La mayoría de los gobiernos a nivel mundial, tienen en sus manos una balanza, en la que en un plato se encuentra la economía (tradicional, clásica, capitalista, esquizofrénica, etc.) y en el otro la salud.

Dada la importancia y urgencia de una economía que sea iluminada por las ciencias de la complejidad, en donde se priorice la VIDA en todas sus manifestaciones; urge y surge la posibilidad y necesidad de la bioeconomía, y con ésta todas aquellas potenciales acciones que ésta conllevaría.

Entre otras acciones se ubicaría la biotributación, término compuesto por "bio", 12 del gr. ßıo- bio- y -ßıos -bios., significa 'vida' $u$ 'organismo vivo', y "tributación", ${ }^{13}$ acción y efecto de tributar (pagar algo al Estado para las cargas y atenciones públicas).

Por consiguiente y como un primer acercamiento al término, se espera que éste apunte a una tributación enfocada en la vida, esto es, en función o en sintonía con la bioeconomía, pero para lograr este tópico es menester repensar la tributación tradicional, pues como ha sido concebida desde sus inicios y como se apuntó antes, deriva de la economía pura o clásica.

Es de anotar que además de los objetivos económicos de los tributos, éstos en la actualidad, también cumplen parcialmente objetivos acordes a la bioeconomía, y en este primer acercamiento se hace referencia a dos potenciales opciones:

La primera, consistiría en elevar o incrementar la carga impositiva, lo que tendría como efecto un desincentivo en la inversión y por ende incrementaría el crecimiento económico negativo (decrecimiento económico), desmotivando el sistema productivo y la comercialización (industrias y empresas), esto es, al mismo capitalismo, el cual como ya se expuso, afecta la vida en todas las manifestaciones posibles, incluyendo la del ser humano, por lo que se coadyuvaría a potencializar, posibilitar, gratificar y exaltar la vida, aportando a la ecuación que propone Carlos E. Maldonado, para el biodesarrollo (crecimiento económico negativo -decrecimiento- por potenciación de la vida).

La segunda y no menos importante, es la que actualmente ejerce la tributación verde en algunos países y que consiste en la regulación de tributos que ayuden directa o indirectamente al medio ambiente, y que según Gemma Patón, citada por Montealegre, Ana Cristina, las razones que justifican esta tributación, y que para efecto del presente artículo, se consideran como más relevantes: "El efecto disuasorio sobre la utilización y el abuso de recursos naturales escasos y sustancias nocivas para el medioambiente; la reducción de los costes en la lucha contra la contaminación, en relación con las políticas medioambientales clásicas; la con-

12 Real Academia Española, "Diccionario de la Academia Española", https://dle.rae.es. Consultado el 3 de mayo de 2020.

13 Idem.

https://revistavidacunori.com 
tribución a acercar los precios de los productos a la "verdad ecológica", considerando los costos externos relacionados con el medioambiente; la posibilidad de simplificar el sistema impositivo eliminando muchos pequeños impuestos que serían sustituidos por los nuevos impuestos ecológicos". ${ }^{14}$

En la mayoría de países, entre estos Guatemala, la tributación verde es una asignatura pendiente por superar, derivado del mismo capitalismo que ha y continúa imperando, por lo que esta tributación se torna utópica, máxime si se le aborda desde las ciencias clásicas, entre éstas la economía pura.

Para ilustrar y evidenciar lo anterior, se recoge una opinión: "Se está poniendo de moda el ecoimpuesto, y en Guatemala hace sus primeras apariciones como "política fiscal verde". Suena bien en teoría, pero en la práctica fácilmente puede caer en la creación de novedosos e ingeniosos mecanismos para extraer más del bolsillo del tributario, obstaculizar la producción(...)",15 en la cual se evidencia, la puntualización de la preocupación por la exacción del tributo, esto es u obedece al objetivo puramente recaudatorio o de asignación de recursos, lo cual se traduce en trasladar la riqueza que obtienen las empresas hacia el Estado y luego éste a la sociedad; así también la preocupación por la obstaculización de la producción, el cual según referimos, es el modelo que permanece intacto a raíz de que el ser humano se ha tornado superior a la naturaleza de la cual se ha apropiado y ha explotado. En suma, se evidencia la preocupación por la afectación del capitalismo, pero en nada por la potencialización de la vida.

\section{Finanzas según las ciencias clásicas}

Las finanzas ${ }^{16}$ se refieren a todas las actividades relacionadas con la obtención de dinero y su uso eficaz. Es importante señalar que las empresas gestionan las formas en que se ha de obtener ese dinero, sin embargo, el campo de mayor acción gira en torno a la correcta utilización del dinero, a ese uso eficaz, ya que, la forma en que se gasta el dinero está condicionada a factores relacionadas al tiempo, al producto, al mercado que se atiende, y al entorno.

Además, las finanzas ${ }^{17}$ estudian la manera en la que la gente asigna recursos escasos a través del tiempo. Dos características que distinguen las decisiones financieras relacionadas con la distribución o asignación de recursos son: 1) se distribuyen a lo largo del tiempo y 2) ni los decisores, ni los demás suelen conocerlos anticipadamente con certeza.

\footnotetext{
14 Montealegre, Ana Cristina, "Desafíos de las reformas fiscales verdes en América Latina. El caso de Nicaragua", Documentos de Trabajo 7/2019. Instituto de Estudios Fiscales, Madrid, España. https://www.ief.es/docs/destacados/publicaciones/documentos_trabajo/2019_07.pdf. Consultado el 30 de mayo de 2020.

15 Fritz Thomas, "La política fiscal verde", Opinión. Prensa Libre, 6 de abril de 2017. https://www.prensalibre.com/ opinion/la-politica-fiscal-verde/. Consultado el 30 de mayo de 2020.

16 Córdoba Padilla, Marcial, "Gestión financiera", ECOE Ediciones. p. 2. Bogotá, Colombia, 2012.

17 Bodie, Zvi y Merton, Robert, "Finanzas", Prentice Hall. p. 2. México, México, 1999.
} 
De lo anterior es importante extraer que el campo de las finanzas la gestión de esos recursos escasos dándole un uso adecuado, un uso eficaz, consiguiendo una maximización en la utilización de los recursos logrando así una mayor rentabilidad.

Lo anterior nos invita a darle un lugar a la palabra decisiones, ya que justamente eso es lo que hacen los administradores financieros, tomar decisiones basadas en experiencias, en informaciones para tratar de ser lo más racional posible. Sabiendo que toda la información que se reúna no le dará certeza, ni le garantizará los resultados por cuanto no se puede conocer anticipadamente los resultados de las decisiones, esto es un nivel de incertidumbre y por cuanto un nivel de riesgo.

\section{La administración o gestión financiera}

La gestión financiera ${ }^{18}$ es un proceso que involucra los ingresos y egresos atribuibles a la realización del manejo racional del dinero en las organizaciones y, en consecuencia, la rentabilidad financiera generada por el mismo. Esto nos permite definir el objetivo básico de la gestión financiera desde dos elementos: la generación de recursos o ingresos, incluyendo los aportados por los asociados; y, en segundo lugar, la eficiencia y la eficacia o esfuerzos y exigencias en el control de los recursos financieros, para obtener niveles aceptables y satisfactorios en su manejo.

Entonces podemos indicar que por un lado las finanzas se encargan de observar y aportar en la generación de los recursos o ingresos (que en economía indicaríamos son escasos), por otro lado, las decisiones que se han de tomar en la asignación de esos recursos a las diversas actividades, velando y exigiendo un uso adecuado y un manejo racional en las erogaciones a través de un estricto control financiero, previamente se hizo un análisis de información (con lo cual se reduce la incertidumbre), ello permitirá decidir entre varias potenciales opciones que encajen en los niveles aceptables y decantarse por aquella que "racionalmente" sea la que aporte mayores niveles de rentabilidad, esto es un futuro y potencial resultado del cual no hay certeza de ocurrencia, por tanto un nivel de riesgo.

A lo anterior hay que agregar que el administrador financiero debe tomar múltiples decisiones, las tres básicas son 1) sobre la inversión, buscando opciones con mayor rentabilidad, menor riesgo y un mayor nivel de liquidez; 2) sobre la financiación, buscando opciones sobre menor costo, tiempo más favorable y menor cantidad de garantías y 3) sobre los dividendos, decidiendo cuanto ha de repartirse o reinvertirse.

18 Córdoba Padilla, Marcial, "Gestión financiera", ECOE Ediciones. p. 2. Bogotá, Colombia, 2012.

https://revistavidacunori.com 


\section{Relación de la economía y las finanzas}

Para establecer las relaciones que existen entre ambas es preciso conocer a que se refiere y que estudia la economía, así economía ${ }^{19}$ es el estudio de la manera en que las sociedades utilizan recursos escasos para producir mercancías valiosas y distribuirlas entre los distintos individuos.

Aunque existen varias cosas por las que se relacionan (la oferta y la demanda que influyen en las finanzas empresariales), nos referiremos a una fundamental, al vínculo común, el dinero, lo que nos lleva al principio de maximizar la utilidad, obteniendo el mayor valor, para debe utilizarse o gastarse la menor cantidad de recursos posible, lo cual está basado en el problema de escases.

Se ha de indicar que la economía en su qué hacer tiene un área de estudio mucho más grande, mientras que las finanzas son una pequeña parte del que hacer económico, su relación para nuestros propósitos tendrá que ver con la gestión del dinero propiamente.

Ahora que tenemos claro el panorama de las finanzas y la economía, así como la relación entre éstas desde las ciencias clásicas, es preciso abordar el tema desde una perspectiva no lineal, más hologramática, por cuánto abordaremos la economía y las desde la perspectiva bioeconomía, para ello es necesario entender qué es y cuál es su campo de acción, así como su variante con la economía propiamente.

\section{Las biofinanzas}

Se ha abordado ya el tema de las finanzas y sabemos que tiene que ver con la buena gestión de los recursos, puntualmente con la administración del dinero, por otro lado, la palabra bio, proviene de la biología, que es la ciencia que estudia los procesos naturales, se cataloga como la ciencia de la vida.

Antes que nada, debemos partir que las biofinanzas son una extensión de la bioeconomía, también debe comprenderse que en ella existen compradores y productores más responsables y más alineados a la naturaleza. Existe consumo responsable y producción siguiendo buenas prácticas y racionalizando recursos, uso recursos renovables, entre otras.

Dado lo anterior supone la existencia de bioactividades (bioturismo, bioenergía, biomateriales, bioinsumos agrícolas, bioquímicos, etc.) que utilizan recursos biológicos (cultivos, ganadería, sector forestal, pesca y acuacultura, recursos genéti-

19 Samuelson, Paul A. y Nordhaus, William, "Economía con aplicaciones para Latinoamérica", McGraw Hill. p. 4. México, México, 2010. 
cos, biomasa y residuos) con lo cual generan bioproductos y bioservicios dentro de las industrias (alimentaria, vestuario y calzado, construcción, química, energía, maquinaria y equipo, farmacéutica y cosmético).

Por lo que necesariamente da lugar a la existencia de los bionegocios, el biocomercio y por su puesto las biofinanzas.

Por lo que una primera definición puede indicar que la biofinanzas son las encargadas de la gestión de los ingresos obtenidos a partir de bio-actividades y que se concentra en la utilización adecuada de los biorecursos en beneficio de la promoción de la vida y la naturaleza, por cuanto existe la capacidad de crear y transmitir valor a la sociedad.

Para poder moldear las actitudes en promoción de las biofinanzas, es necesario también abordar las neurofinanzas y entender la irracionalidad que existe en los seres humanos y lo que lo condiciona en el momento que toma decisiones financieras.

\section{Las neurofinanzas}

Las finanzas clásicas asumen que la gente que compone los mercados y las sociedades actúan siempre de forma racional, esto es porque, éstos basan sus decisiones en la información disponible previamente analizada, lo que no se podía explicar era los patrones de comportamiento y conducta de los individuos ante ciertos eventos que no eran tan racionales y que influían en las decisiones.

Para poder comprender de manera más integral y más profunda las fianzas debe entenderse que éste tiene inherentemente un componente psicológico, por lo que de la aplicación de las ciencias conductuales a las fianzas da como resultado la neurofinanzas, estas son la psicología, neurociencia cognitiva y la sociología. Con lo que la unión de todas estas ciencias se podría explicar cómo ciertos rasgos, ciertos comportamientos y su entorno influyen en las personas que toman decisiones a pesar de la información que éstos posean. "Así podemos indicar que la neurofinanzas estudian la naturaleza de los procesos cognitivos que participan en la adquisición y el procesamiento de información en la toma de decisiones financieras." 20

20 Girón, Natalia, El blog de las finanzas.11/10/2018. https://elblogdelasfinanzas.wordpress.com/2018/10/11/ que-son-las-neurofinanzas/. Consultado el 26/05/2020. 


\section{"Dos sistemas cognitivos}

Los psicólogos dividen nuestro pensamiento cognitivo en dos sistemas diferentes: el sistema inconsciente (de pensamiento rápido y que ésta en primer plano) y el sistema consciente (de pensamiento lento y que ésta siempre en espera)." 21 "Gran parte de la actividad neuronal del cerebro ocurre sin que seamos plenamente conscientes de ello, de hecho, el $95 \%$ de nuestro comportamiento se realiza de forma inconsciente".22

El sistema inconsciente está ligado a los sentimientos y a las emociones, es de respuesta rápida, lo que provoca que las decisiones tomadas no sean analizadas y por ende no son cruzadas con información que permita minimizar el riesgo.

El sistema consciente está ligada a la razón, es de respuesta lenta, ya que las respuestas han sido analizadas, lo que permite minimizar el riesgo. Es por ello que se dice que las decisiones tienden a tomarse más con el corazón que con la razón.

\section{Las biofinanzas y la bioeconomía}

Ante un mundo de hiperconsumismo, rienda suelta a las emociones de compra en donde predomina el ego y el individualismo, pre supone la supremacía del consumismo, en donde cada vez son más escasos los recursos, la capacidad regenerativa del planeta es menor y que a menudo la ocurrencia de eventos naturales provocan desastres y caos, el más reciente el COVID-19, lo que pone entre dicho la forma normal de comportamiento y provoca que se volteen a ver nuevos modelos o nuevas formas de comportamiento.

Ante tal panorama la bioeconomía ofrece una salida a la economía tradicional por tanto al sistema capitalista, esto es poseer compradores responsables en el consumo, así como en los hábitos de compra, tomando un pensamiento más colectivo y menos individualista, con ello la racionalidad es obligatoria, buscando además alternativas de origen natural, es allí donde entran los productores con un enfoque honesto, cooperativo que busca proveer al mercado soluciones naturales, ecológicas y que han sido elaboradas usando mecanismos no contaminantes, usando energías limpias y renovables, además del uso racional de los biorecursos.

En tal premisa, los consumidores y productores son responsables con el Estado brindando a través de la biotributación los ingresos que el éste requiere para cum-

\footnotetext{
21 Franch, Miriam. Rankia, 27/05/2016. https://www.rankia.com/blog/fondos-inversion/3230666-neurofinanzas-como-toma-cerebro-decisiones-financieras. Consultado el 27/05/2020.

22 Escalona, Pablo, Declarando. 25/07/2018. https://www.autonomosyemprendedor.es/articulo/tu-negocio/95-decisiones-compra-son-inconscientes/20180724141759017163. Consultado el 26/05/2020.
} 
plir con sus obligaciones las cuales buscan generar un biodesarrollo y por cuanto un bienestar generalizado con acceso equitativo e igualitario para la población de los servicios necesarios para tener una vida digna, no llena de lujos, los cuales tienden a desaparecer producto de un pensamiento más racional.

Justo allí es donde tiene cabida las biofinanzas las cuales son capaces de administrar correctamente los recursos, mediante la captación de ingresos provenientes de actividades limpias y que son asignadas racionalmente atendiendo el estado irracional de la persona en forma tal que se asegure la sostenibilidad de las actividades bioeconómicas.

Para lograr tales actividades será fundamental la intervención de la bioética que logrará convencer a los humanos irracionales de las premisas equivocadas para poder lograr un trabajo colaborativo de una manera más equitativa, con los beneficios que conlleva en el desarrollo común producto de decisiones colectivas y no individualistas, entre eliminar el ego y el aprovechamiento en pro de un beneficio colectivo.

\section{Bioética: una supra ciencia en la época de los coronavirus}

EI SARS-CoV-2, el coronavirus que provoca el COVID-19, es posiblemente la gota que rebalsó el vaso. O la última llamada de atención a la conciencia de la humanidad. COVID-19 es un acrónimo propuesto por la Organización Mundial de la Salud (OMS), compuesto por COrona + Disease (en idioma Inglés) + [20] 19. No obstante, otra interpretación (personal) puede ser: el prefijo latino co (con sus variantes con, com, col o cor), que deriva del latín cum que significa agregación, unión o compañía, y de allí se construye colega, compañero, cooperar, corromper, coaligar, etc. Y vid, que iserá vida, o al menos tendrá un eco de ella? En otras palabras -en nuestra interpretación libre-, mantener la vida nos va en conjunto: covid, "la vida compartida". Se salva la especie, no los individuos. Y para eso es requisito que quienes detentan el poder en el mundo lo cedan a la ciudadanía universal. Se hace imprescindible una nueva ética, una bioética que sea coherente con lo que predican los grandes sistemas (que son o pretenden ser) espirituales; o bien, los devenidos en religiones. El ser humano requiere dejar de competir y genuinamente empezar a cooperar. Desterrar de su mente que la naturaleza le pertenece y que está a su servicio. Más, que dialogue respetuosamente con ella. El insight, cualquier manifestación de vida es, por decir lo menos, sagrada. 


\section{De la ecoética a la bioética}

A lo largo de su devenir histórico la humanidad y las categorías genéticamente hermanadas (neandertales, denisovanos, etc.), se han enfrentado a desafíos formidables que han ubicado en el espacio del riesgo su continuidad como especie. Quizá la prueba de ello sea que -hasta donde se sabe- en la actualidad sólo sobrevivamos los sapiens sapiens; si bien las desparecidas siguen presentes a través de los genes; las unidades vitales -como se sabe-, que en efecto se replican y reproducen. Y por lo tanto se perpetúan.

En la historia de la humanidad es muy probable que no se encuentren períodos relativamente largos (cuantificados según su métrica inherente, más allá de los años que en promedio se dilata una vida cuya expectativa cada vez dura más), en los cuales se diga que se ha vivido en armonía. Como sucede en la Utopía de Thomas Moro, en La República de Platón, o demás ciudades fantásticas retratadas en obras clásicas. Con seguridad y optimismo; con libertad, cobijo, juego y alimento; con genuina esperanza en el futuro. En paz y sin ansiedad, incertidumbre o temor hacia el devenir. Que deje en la mitología al célebre tigre diente de sable, al cual se le endilgan muchas de nuestras taras.

Hasta antes de que finalizara la conflagración denominada Segunda Guerra Mundial, todavía era frecuente que sucedieran catástrofes naturales que podían impactar a cualquier región del mundo, como las recurrentes hambrunas. Aún a principios del siglo 20 las fotografías de la época evidencian que por ejemplo la desnutrición no era ajena a todas las clases sociales. Además, por si no fuera suficiente con la promesa del más allá, como una justificación o dulce expiación, había enfermedades causadas o asociadas a las carencias familiares, a las limitaciones económicas, a la pobreza tal cual, a la vulnerabilidad social y a las difíciles condiciones de vida, como el caso de la tuberculosis o tisis que se consideraban incluso como una especie de privilegio o hasta de "refinamiento". La época de gloria del Romanticismo.

Todavía la industrialización y la tecnología que conocemos hoy en el mundo no eran lo que llegarían a ser: Una solución (al menos aparente) para una cierta proporción de sus sociedades (las clases altas y en menor medida las llamadas clases medias). Mas no para el resto de la humanidad, especialmente para aquellas regiones que se dice han sido "olvidadas incluso por dios".

Sabemos con certeza que las pestes, las hambrunas, la pobreza, la miseria, y la iniquidad del hombre contra el hombre nunca han sido eliminadas y ni siquiera atenuadas de la faz de la Tierra. Son arcanas taras omnipresentes. No obstante, gran parte de la humanidad parecía -o incluso hoy en día parece- no haberse dado cuenta del rumbo que tomaba la vida en el planeta. La superestructura 
ideológica, a través de la publicidad, de la propaganda, de las relaciones públicas, de la guerra psicológica, de la desinformación, empleando los mass media, etc., han hecho muy bien su trabajo: "esclavos defendiendo la esclavitud".

La crisis del cambio climático y el consecuente calentamiento global, la inexistente realidad de un desarrollo sostenible a nivel mundial, o más bien de ninguna clase de desarrollo en el resto del mundo, auspiciaban que la humanidad y el resto de la vida no se conducían hacia un puerto tan siquiera medianamente seguro. Es un virus, una entidad carente de vida en el estricto sentido del concepto, lo que vino a sacudir a la humanidad, a sacarla de su alienado marasmo y a permitirle comprender que una partícula microscópica podía ser capaz de hacer tambalear a la ufana civilización humana. No sin, por supuesto, la colaboración de las tan minúsculas como poderosas élites que dominan el rumbo de la humanidad y del planeta.

Se ha vuelto un lugar común -pero no por ello menos cierto- el hecho de que el mundo conocido aún a inicios de marzo de 2020 nunca volverá a ser lo que fue. Un mundo de ensueño y fantasía, un paraíso en este mundo para menos del dos por ciento de la humanidad, o una realidad abyecta y carente de futuro para el abrumador restante porcentaje. Empero, una considerable fracción de éstos creía vivir en un estado transitorio, una especie de limbo, que su estatus era temporal y que por arte de magia algún día pasaría a formar parte del selecto club de privilegiados del mundo, aquellos que detentan por sí y ante sí más de la $80 \%$ de la riqueza, relegando la quinta parte residual para que se distribuya entre el $98 \%$ disperso en prácticamente todo el planeta.

La duda actual es ¿̇hacia dónde se conduce la humanidad? ¿̇Hacia un nuevo amanecer más congruente con lo que se considera que merece el ser humano? $\dot{\imath} \mathrm{O}$ a una sociedad totalitaria, un estado policial completamente controlado y cooptado, con grandes masas de individuos desprovistos de autonomía, libertad efectiva y voluntad; manipulados cual autómatas o zombis?

Cruentos ejemplos de esta última realidad existen desde la famosa noche de los tiempos. Conocemos suficientemente atisbos de lo que podría ser una sociedad planificada, programada, organizada y controlada hasta extremos demenciales. La distopía.

El otro caso, el tránsito hacia una sociedad congruente con lo que predican muchos sistemas religiosos o espirituales, requiere del nacimiento de una nueva ciencia integrativa, global e inclusiva. Una ciencia humana y del ser humano que lo incluya en todas sus dimensiones. En lo material y en lo espiritual. En lo individual o particular tanto como en lo social y colectivo. Esa ciencia no podría ser otra más 
que una ética que trascienda lo inmediato, lo coyuntural, lo particular, todas las prácticas en fin que han arrastrado a la humanidad a este deplorable e inhumano estado de cosas que de sobra conocemos.

Es una ética que coloque como núcleo la vida. La vida en todo su esplendor. No la vida como la ha conocido la humanidad en la hora casi 24 del Reloj de Sagan. Una ética que de forma temporal llamaremos bioética, por carecer de un mejor término y mientras surge uno que trascienda lo meramente biológico, lo de las ciencias médicas, lo de la vida material como un fenómeno producto de la probable combinación fortuita de factores o elementos químicos a determinadas condiciones de presión y temperatura.

Una ética que incorpore los valores más excelsos que ha creado el cerebro o el espíritu humano. Una ética que incorpore la incertidumbre, la complejidad, lo holográfico. Un salto cuántico real en lo humano que a manera de propiedad emergente en primer lugar detenga el pronunciado deterioro de la biósfera y a partir de allí se inicie la verdadera edad de oro de la humanidad. Quizá el tan citado eterno retorno de las sociedades herméticas.

En la hora 23:59:55 del Reloj de Carl Sagan la humanidad no había alcanzado las cotas actuales. Hoy, con la Teoría de la Relatividad comprobada, con la Mecánica Cuántica no comprendida pero sí ampliamente aplicada, con el notable desarrollo científico y tecnológico en indetenible ascenso, como lo demuestran la neurociencia, la robótica, la inteligencia artificial, la big data, el teletrabajo, la algoritmia, la cibernética; de las empresas que facturan cifras mayores que el producto interno bruto de muchos países de lo antiguamente denominado Tercer Mundo.

No deja de ser una dolorosa paradoja que contando prácticamente con las condiciones ideales para alcanzar lo que se puede considerar una o la gran convivencia y fraternidad universal humanista, más bien pareciera que estamos ante una nueva versión de la búsqueda del punto de origen del arcoíris. O en la incesante persecución del para nada ubicuo horizonte, que conforme más avanzamos más lejos nos encontramos. O la noria.

Si cabe, es notable que la humanidad en la práctica ha avanzado exponencialmente en todos los órdenes; excepto en lo espiritual. Se ha incrementado la expectativa de vida a casi el doble de hace menos de cien años. Se pueden producir alimentos sin sacrificar al planeta para satisfacer las necesidades de alimentación de los casi 8 millardos de estómagos. No obstante, hay marginación, desigualdad, exclusión, segregación, discriminación, relegamiento, desnutrición, vida indigna en fin para la humana especie. 
La humanidad va a reescribir la filosofía y a corregir gran parte de lo que se consideraba conocimiento consagrado. Mas quién dirigirá el cambio que la humanidad no puede posponer so pretexto de carecerse de otras opciones.

Sólo a través de la transdisciplinariedad, de la aplicación certera del pensamiento complejo y de todo el herramental que con no poco esfuerzo se ha desarrollado por parte de individuos que al sintetizar el producto social alcanzaron a ver que más allá de las apariencias o de lo que considerábamos era "la verdad", existe una realidad que podemos construir. Una nueva Ética. Un desafío ineludible e impostergable.

\section{Reflexiones}

¿Pudiésemos asentar que el hombre, en el transcurrir de los tiempos, no ha evolucionado sino involucionado?, pues ha destruido su propio hábitat y a él mismo.

Lo anterior se ha evidenciado a lo largo de la historia con varias crisis ambientales, económicas y sanitarias, pero a hoy, con mayor razón, con la aparición y propagación del Coronavirus (COVID-19).

Hoy más que nunca se pone de manifiesto la necesidad de abordar la economía desde las ciencias de la complejidad, esto es, la bioeconomía y lo que ésta conlleva, que entre otras opciones, se tiene al biocomportamiento para la correcta toma de decisiones a favor de la vida; la biotributación, que sería una forma diferente, contrastante y desafiante de comprender y aplicar los tributos, cuyos principales objetivos apuntarían a propiciar el decrecimiento económico, desincentivar el capitalismo, el hiperconsumismo y formentar el consumo responsable; así también coadyuvar y promover la protección del medio ambiente mediante la tributación verde o ecotributación. Todo esto podría tener un efecto en la disminución de necesidades y deseos de la sociedad, por lo cual sería poco o nada necesario contar con recursos públicos para su satisfacción.

Finalmente, podría pensarse que si desde algún tiempo atrás, se hubiese aplicado la bioeconomía coadyuvada por la biotributación, quizá no padeciésemos (o padeciésemos a menor escala) las crisis que hoy nos aquejan (ambientales, económicas y sanitarias) y el Coronavirus (COVID-19) no estaría en su máxima expansión y propagación a nivel mundial. Además, las biofinanzas que promueven la adecuada utilización de los recursos financieros a favor de la vida, considerando como un elemento de incalculable valor a la bioética, responsable de que las decisiones sean coherentes e íntegras en su plenitud. 


\section{Referencias Bibliográficas}

Bodie, Zvi y Merton, Robert, "Finanzas", Prentice Hall. México, México, 1999.

Cantú Martínez, Pedro César, "Bioética e investigación en Salud", Trillas Tercera Edición, México, 2015.

Castillo López, Nancy Carolina, "El fenómeno del Coronavirus y el Biodesarrollo: sus consecuencias y oportunidades", Revista Ciencia Multidisciplinaria CUNORI Vol. 4, No. 2, USAC, Guatemala, 2020, https://doi.org/10.36314/cunori.v4i2.136

Córdoba Padilla, Marcial, "Gestión financiera", ECOE Ediciones, Bogotá, Colombia, 2012.

Delgado Díaz, Carlos Jesús, "Hacia un nuevo saber. La bioética en la revolución contemporánea del saber", Universidad El Bosque, Primera Edición, Colombia, 2015.

Fritz Thomas, "La política fiscal verde", Opinión. Prensa Libre, 6 de abril de 2017. https://www.prensalibre.com/opinion/la-politica-fiscal-verde/. Consultado el 30 de mayo de 2020.

García Romero, Horacio y Luis Limón Limón, "Bioética General", Trillas, Tercera Edición, México, 2018.

Girón, Natalia, "Blog de las finanzas", https://elblogdelasfinanzas.wordpress. com/2018/10/11/que-son-las-neurofinanzas/. Consultado el 26/05/2020.

Guirola López, José Manuel \& Martín Román, Javier, "Economía, Hacienda Pública y Política Fiscal. Tema 1: El Marco Económico Internacional. Macromagnitudes e Indicadores Macroeconómicos. Hacienda Pública y Fiscalidad: Teoría de la Imposición", Universidad Nacional de Educación a Distancia (UNED) e Instituto de Estudios Fiscales (IEF). Madrid, España, 2017.

Kliksberg, Bernardo, "Más ética más desarrollo", Temas, Cuarta Edición, Argentina, 2005.

Llarandi Arollo, Carlos, "Tres principios del pensamiento complejo. La búsqueda de la verdad es una auténtica vocación del ser humano", En: Https://profesionalesporelbiencomun.com/tres-principios-del-pensamiento-complejo/\#.XrGo96hKjlU. Consulta realizada el 01 de mayo de 2020.

Maldonado, Carlos Eduardo, "Bieconomía, ciencia y lenguaje", Entrevista: https:// www.youtube.com/watch? $v=c M d 44 T P Q 6 X M$. Consultado el 3 de mayo de 2020. 
Maldonado, Carlos Eduardo, "Complejidad de las ciencias sociales. Y de otras ciencias y disciplinas", Ediciones desde abajo. p. 202. Bogotá, D.C., Colombia, 2016.

Maldonado, Carlos Eduardo, "¿Qué significa la crisis del Coronavirus?", https:// www.desdeabajo.info/ediciones/item/39464-que-significa-la-crisis-del-coronavirus.html. Consultado el 3 de mayo de 2020.

Montealegre, Ana Cristina, "Desafíos de las reformas fiscales verdes en América Latina. El caso de Nicaragua", Documentos de Trabajo 7/2019. Instituto de Estudios Fiscales, Madrid, España. https://www.ief.es/docs/destacados/publicaciones/documentos_trabajo/2019_07.pdf. Consultado el 30 de mayo de 2020.

Rankiahttps://www.rankia.com/blog/fondos-inversion/3230666-neurofinanzas-como-toma-cerebro-decisiones-financieras. Consultado el 27/05/2020.

Real Academia Española, "Diccionario de la Academia Española", https://dle. rae.es. Consultado el 3 de mayo de 2020.

Rosa Jeovany, Jack Farrington, Edwin Franco, Mayra Pinto, Rodolfo Compá, "Dialogando con la Sociedad sobre Complejidad, Educación Salud y Vida", Revista Vida una Mirada Compleja Vol. 1, No. 1, p. 29. CUNORI. Guatemala, 2019. https:// doi.org/10.36314/revistavida.v1il.1

Ruiz de Chávez, Manuel, (Coordinador) "Bioética y Derechos Humanos XXV años de reflexiones", Fontamara, Primera Edición, México, 2018.

Ruiz de Chávez, Manuel, (Coordinador) "Temas selectos de conbioética. Actualidades y perspectivas", Fontamara, Primera Edición, México, 2018.

Samuelson, Paul A. y Nordhaus, William D., "Economía con aplicaciones para Latinoamérica", McGraw Hill. México, México, 2010.

\section{Biografía de los autores}

Estudiantes del Doctorado en Investigación en Educación en el Centro Universitario de Oriente CUNORI de la Universidad de San Carlos de Guatemala. 
Los textos publicados son responsabilidad de los autores. Copyright @ 2 2020. Los derechos son de los autores. Oscar Eduardo Orellana Aldana, Axel Esaú Brenes Villeda, Adery Luciano Pérez Yoc y Sergio Giovanni Gatica.

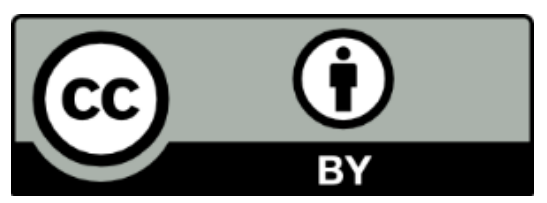

Los textos están protegidos por una licencia Creative Commons 4.0 Internacional Usted es libre de compartir, copiar y redistribuir el material en cualquier medio 0 formato y adaptar el documento, remezclar, transformar y crear a partir del material, siempre que cumpla con la condición de atribución, debe reconocer el crédito de la obra de manera adecuada.

\section{El manuscrito es de acceso abierto}

